\title{
AN EMPIRICAL METHOD FOR FAST PREDICTION OF RAREFIED FLOW FIELD AROUND A VERTICAL PLATE
}

\author{
HE TAO \\ College of Aerospace Engineering, Nanjing University of Aeronautics and Astronautics \\ Nanjing, Jiangsu Province, 210016, China \\ nuaahetao@126.com \\ WANG JIANG-FENG \\ Hypersonic Aerodynamics Research Center (HARC), Nanjing University of Aeronautics and Astronautics \\ Nanjing, Jiangsu Province, 210016, China \\ wangj@nuaa.edu.cn
}

Published 22 June 2016

\begin{abstract}
Numerical study is conducted to investigate the effects of free-stream Knudsen $(K n)$ number on rarefied flow field around a vertical plate employing an unstructured DSMC method, and an empirical method for fast prediction of flow-field structure at different $K n$ numbers in a given inflow velocity is proposed. First, the flow at a velocity $7500 \mathrm{~m} / \mathrm{s}$ is simulated using a perfect-gas model with free-stream $K n$ changing from 0.035 to 13.36 . The flow-field characteristics in these cases with varying $K n$ numbers are analyzed and a linear-expansion phenomenon as a function of the square of $K n$ is discovered. An empirical method is proposed for fast flow-field prediction at different $K n$ based on the least-square-fitting method. Further, the effects of chemical reactions on flow field are investigated to verify the applicability of the empirical method in the real gas conditions. Three of the cases in perfect-gas flow are simulated again by introducing five-species air chemical module. The flow properties with and without chemical reactions are compared. In the end, the variation of chemical-reaction flow field as a function of $K n$ is analyzed and it is shown that the empirical method are also suitable when considering chemical reactions.
\end{abstract}

Keywords: $\mathrm{Kn}$; rarefied flow; DSMC; flow-field structure; empirical method; chemical reactions

\section{Introduction}

With the rapid development of hypersonic vehicle technique, many researches are focused on the rarefied flow regime, where continuous medium hypothesis is no longer valid and a micro molecular model needs to be constructed. The studies should be carried on through the methods of the rarefied gas dynamics ${ }^{1}$.

According to the gas rarefaction which is judged by the free-stream Knudsen number $(K n)$, rarefied flows are divided by $\mathrm{Tsien}^{2}$ into three regimes: the slip flow regime $(0.01<K n<0.1)$, the transitional flow regime $(0.1<K n<10)$ and the free-molecular flow regime $(K n>10)$. And $K n$ is the ratio of the mean free path $\lambda$ and the characteristic

This is an Open Access article published by World Scientific Publishing Company. It is distributed under the terms of the Creative Commons Attribution 3.0 (CC-BY) License. Further distribution of this work is permitted, provided the original work is properly cited. 
length $L$. To solve the aerodynamic problems in rarefied regime, $\operatorname{Bird}^{3-4}$ proposed the Direct Simulation Monte-Carlo method (DSMC) in 1976, which is proved to be a very useful approach to simulate the rarefied gas flows and considered as the representative achievement in the late 20 th century ${ }^{5}$.

In the present researches on rarefied flows, much attention is paid to the aerodynamic properties of hypersonic aircrafts ${ }^{6-12}$ such as the force and the heat transfer. Potter et al. ever gave a computational method for the prediction of aerodynamic forces and moments of axi-symmetric hypersonic bodies in the transitional flow region according to data from numerical simulation and experiment ${ }^{12}$. Moss presented variations of drag and heating coefficients as a function of rarefaction when simulating ballute's aerodynamics ${ }^{9}$. Ozawa et al. found a bridging function to represent the aerodynamic characteristics of Mars explorer by carrying out a series of numerical simulations from the free-molecular flow to the continuum flow ${ }^{11}$. However, in these researches, variations of flow-field properties affected by gas rarefaction get little attention; neither do we have any empirical engineering method to predict the flow-field structure at different $K n$ rapidly.

In this paper, the DSMC method based on the unstructured meshes technique is used to study the effects of $K n$ on hypersonic rarefied flow properties. Taking both the perfectgas and the chemical non-equilibrium flow around a vertical plate into consideration, the variations of rarefied flow-field structure versus $K n$ in a given velocity are investigated in detail. Through the flow-parameter-tracking technique and the least-square-fitting method, an empirical method used for fast prediction of flow-field structure at different $K n$ is proposed. In a given-inflow-velocity condition, it is only needed to calculate rarefied flows at two Knudsen numbers and the flows in other Knudsen number condition can be predicted directly using the empirical method, instead of numerical simulation respectively.

\section{DSMC Method}

In this paper, all simulations are performed using a DSMC code developed by our team from the Hypersonic Aerodynamics Research Center (HARC) in Nanjing University of Aeronautics \& Astronautics (NUAA) ${ }^{13}$. The DSMC program is based on unstructured mesh technique, including the general computational models proposed by Bird ${ }^{4}$. After so many years' development, maintenance and validation ${ }^{13}$, the DSMC code is proved to be stable, robust and has a high efficiency.

Several important models and techniques in the simulations are chosen as follows: (1) Maxwell diffuse reflection model is used for gas-surface interaction; (2) surface temperature is supposed to be constant; (3) molecular mutual collisions are simulated using the variable hard sphere (VHS) model; (4) for possible collision partners selection, the no-time-counter (NTC) scheme is adopted, which decreases the requirement of algorithm to the mesh scale; (5) the energy exchange between kinetic and internal modes is controlled by the Larsen-Borgnakke statistical model; (6) five-species $\left(\mathrm{O}_{2}, \mathrm{~N}_{2}, \mathrm{O}, \mathrm{N}\right.$, NO) reacting air gas model is adopted with 23 chemical reactions ${ }^{14}$, including dissociation, exchange and recombination; (7) for a fast and load-balancing computation, 
a MPI-parallel algorithm of the unstructured DSMC method is used with an adaptive domain decomposition technique introduced.

\section{Analysis of $K n$ effects on perfect-gas flow}

In this section, the effects of $K n$ on rarefied flow in perfect-gas condition are studied. To save the computational cost, a vertical-thin plate is chosen as the geometry. The freestream Knudsen number is modified through changing inflow molecular number density $n$, while the plate's length is kept constant.

\subsection{Free-stream conditions and computational grids}

The length $L$ of the plate is $4 \mathrm{~m}$ and the thickness $t$ is $0.05 \mathrm{~m}$. Free-stream conditions are listed as follows. The flow direction is vertical to the plate; the velocity $V_{\text {inf }}$ and temperature $T_{\text {inf }}$ are $7500 \mathrm{~m} / \mathrm{s}$ and $240 \mathrm{~K}$, respectively; the wall temperature $T_{\text {wall }}$ is kept $500 \mathrm{~K}$; the initial mole fractions of oxygen molecule and nitrogen molecule are 0.75 and 0.25 . The molecular number density $n$ is taken from the atmosphere altitude from $100 \mathrm{~km}$ to $160 \mathrm{~km}$ at $5 \mathrm{~km}$ interval (refer to U.S. 1976 Standard Atmosphere Model ${ }^{15}$ ); corresponding free-stream Knudsen numbers (based on the length $L$ of the plate) are respectively $0.035,0.084,0.197,0.436,0.83,1.4,2.19,3.22,4.53,6.16,8.14,10.53$ and 13.36. There are totally 13 simulation cases changing from the slip flow regime to freemolecular flow regime. Computational grids are generated using the Pointwise software and the scales of cells are based on the mean free path. Taking the first four cases for example, the whole computational region is a square with a $10 \mathrm{~m}$ length side, the cell number are about 560000, 220000, 140000 and 30000, and the simulated particle number are about 14, 5.3, 3.2 and 0.7 million. Figure 1 shows the partial meshes around the plate of the four cases.

All the simulations are run on a 16-node Linux PC-cluster system. The calculation time is determined by simulated molecular number. For the case $K n=0.035$, it's about 72 CPU hours; for $K n=0.436$, it's about 8 CPU hours.
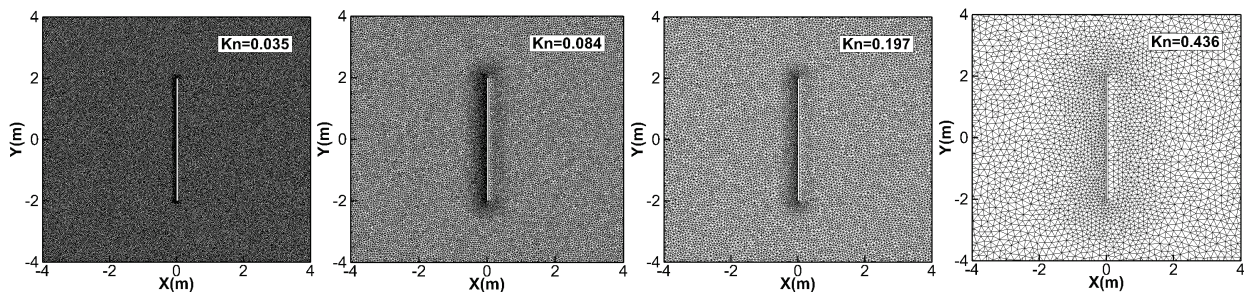

Fig. 1. Partial unstructured meshes at different $K n$ numbers.

\subsection{Analysis of flow field}

Fig. 2 presents velocity contours in flow field at different $K n$ number under perfect-gas flow conditions. As we can see, for the case $K n=0.035$ which is in the slip regime, a strong detached bow shock is developed starting from the front of the plate and extending 
to both sides symmetrically. In the shock wave region, velocity descends sharply from a hypersonic value to subsonic. Along with $K n$ increasing, the flow is close to the transitional regime. It could be seen that the disturbed flow field gradually expands outward and the shock layer becomes thicker, while the detached distance larger and the shock intensity weaker. When $K n$ comes up to some value, for example $K n=0.436$, the shock wave almost disappears and the velocity in flow field diminishes slowly from the free-stream value to zero on the surface instead of sharply falling-down. In the meanwhile, the inner flow field structure shrinks close to the plate's two ends, and a large arc-expansion region is formed in the windward side of the plate. When $\mathrm{Kn}$ rises continually, it belongs to the transitional flow regime and free-molecular flow regime. It could be seen from the bottom four pictures in Fig. $2(K n=1.4 \sim 10.53)$ that all cases have a similar topology flow-field structure, which seems like a clenched fist. The main differences are that the whole disturbed flow-field is larger and larger due to gradual expansion.

The reason for this phenomenon could be explained as follows. When free stream is close to the continuum regime, the gas has so large molecular number density that molecules reflected by the plate will suffer quantities of collisions with inflow molecules. The result of collisions between the inflow and reflected molecules is the formation of shock layer as the neutral position. Inflow molecules with a hypersonic velocity slow down through the shock layer and molecular kinetic energy transfers to internal energy. At the same time, reflected molecules' velocity also diminishes and changes so much that they can't go across the shock layer to far field. Thus, disturbance induced by the plate is restricted between the plate and the shock layer in the windward region and can't spread forward too far. However, when gas rarefaction increases, molecular number density falls down. Reflected molecules can't experience enough collisions with inflow molecules, and the intensity of collisions becomes weak. It means that molecules when reflected by the wall can arrive at a farther position in flow field in front of the plate. The shock wave which plays a role of neutral balance will be pushed away from the wall, with the strength weakened and the layer thicker. Then it may disappear in some rarefaction situation. In the meanwhile, the resistance to forward-propagation of the disturbance becomes weaker and weaker. So the area of disturbed flow field becomes larger. As shown in Fig. 2, flow field dilates along with $K n$ increasing. As for the arc flow structure in front of the plate, it can be attributed to the density gradient existing between the wall surface and the far field.
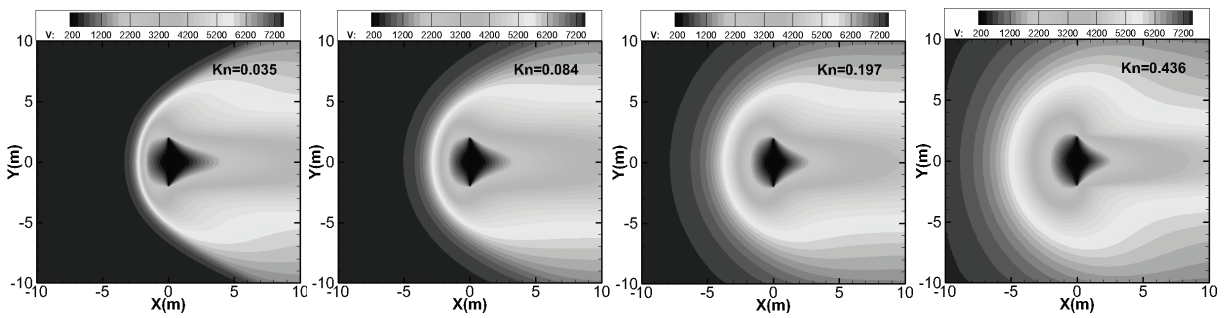

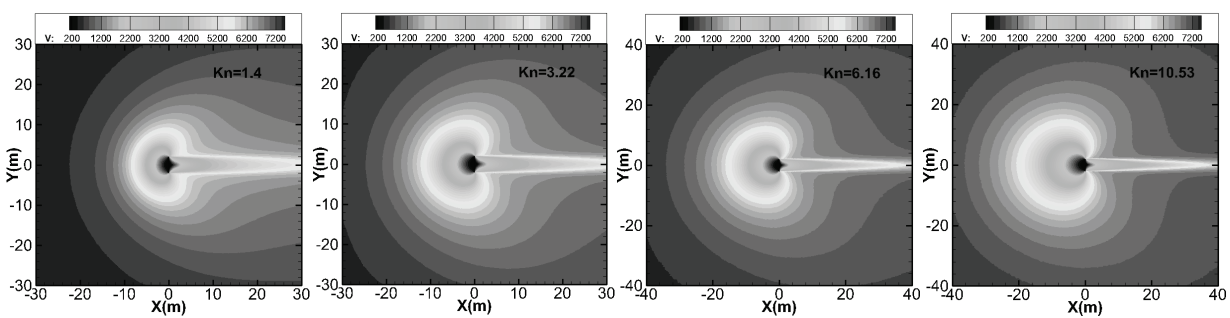

Fig. 2. Velocity contours vs. $K n$ under perfect-gas flow conditions.

Figure 3 illustrates flow properties along the stagnation line with $K n$ changing from 0.035 to 10.53 , including velocity and density. As shown in Fig. 3a, velocity in these cases all falls down along the stagnation line in front of the plate. However, the gradients are different. For little Knudsen numbers, it descends more sharply meaning a thinner shock layer; with $K n$ larger, it changes more gently indicating that the shock layer becomes thick; for large Knudsen numbers, it almost decreases at an approximately average gradient showing that shock wave is unapparent. In the leeward region, the velocity goes up again.

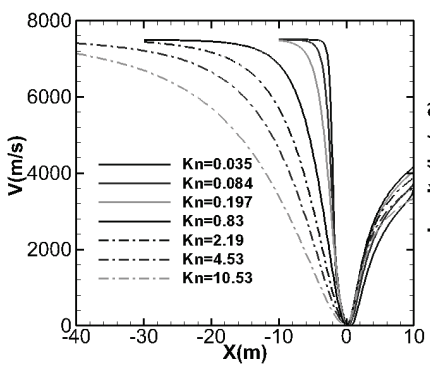

a. velocity

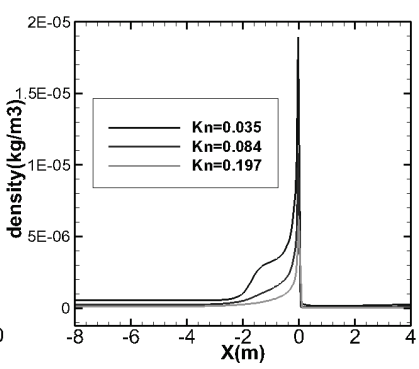

b. density $(K n=0.035 \sim 0.197)$

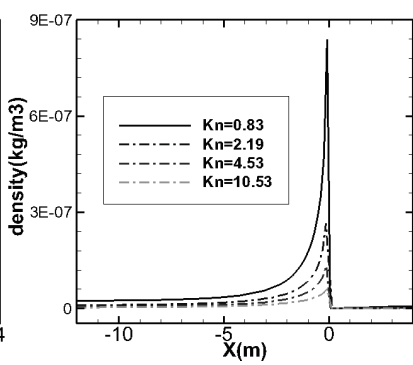

c. density $(K n=0.83 \sim 10.53)$

Fig. 3. Flow properties along the stagnation line.

Because flow parameters are calculated with dimension and density values in these cases may have a difference of several orders of magnitude, density distribution is presented in Figs. 3b and 3c. It can be seen that density in front of the plate rises along the stagnation line in all the cases. And the mass compression ratio of wall surface density to far-field reaches 30 at most. An especially obvious phenomenon is that the increase of density in the case $K n=0.035$ is composed of two stages, which are located in the shock wave region and the thermal boundary layer respectively. The reason for the first rise stage is that the shock wave is so strong and thin that inflow gas is compressed sharply when across that region. The result agrees well with Bird's numerical simulation to one-dimensional steady flow using DSMC method ${ }^{15}$. However, there is only one-rise stage in the stagnation line in the other cases, which is in the thermal boundary layer. The reason could be explained as follows. When $K n$ rises, the intensity of shock wave fades and the thickness increases. The compression effects on gas molecules are too weak to be ignored. The thermal boundary layer becomes thick along with flow field's expansion 
and intersects with the shock wave region. As a result, two-rise stages are replaced by only one-rise stage.

\subsection{Establishment of a flow-field prediction method}

According to the researches in subsection 3.1 and 3.2, it's known that flow field expands outward along with $K n$ increasing and some regular characters are shown during the variation course versus $K n$. Inspired from the analysis of flow properties distribution in Fig. 3, the variation regular characters can be presented through marking a flow parameter and tracing its position.

In this paper, a dimensionless position $D^{*}$ is designed as the marker of flow field. It is defined by the position of a given velocity in the front stagnation line. The distance $D$ of the position to the plate is measured and $D^{*}$ is obtained by dividing the characteristic length $L$, that is, $D^{*}=D / L$.

In this section, two velocity $70 \% V_{\text {inf }}$ and $50 \% V_{\text {inf }}$ in the stagnation line are selected and the corresponding dimensionless positions are labelled as $D_{1}^{*}$ and $D_{2}^{*}$, respectively. Fig. 4a shows the distribution of $D^{*}$ in all the cases. As seen in the picture, the variation curve of $D^{*}$ as a function of $K n$ is close to a parabola with the symmetric parallel with Xaxis, no matter for $70 \% V_{\text {inf }}$ marked position or $50 \% V_{\text {inf }}$ marked position. It's indicated that flow field expands outward at a parabolic tendency with the Knudsen number rising.

To investigate the relationship between $D^{*}$ and $K n$, a fitted line of $D^{*}$ as a function of $\sqrt{K n}$ is plotted based on the least square method. As presented in Fig. $4 \mathrm{~b}, D^{*}$ has a highly linear increase with $\sqrt{K n}$ rising and points are almost completely distributed on the fitted line in the two marked positions. Based on the variation law, we define the relationship between $D^{*}$ and $K n$ in perfect-gas flow as a linear function $D^{*}=\mathrm{a} \sqrt{K n}+b$ in this paper, where a and $b$ are respectively the slop coefficient and intercept coefficient of the line. For the two marked positions, the coefficients are respectively $a_{1}=1.2307$, $\mathrm{b}_{1}=0.3384$ and $\mathrm{a}_{2}=0.7508, \mathrm{~b}_{2}=0.3468$.

As a conclusion, it is indicated by researches in this section that flow field expands linearly with $\sqrt{K n}$ increasing under perfect-gas flow conditions.

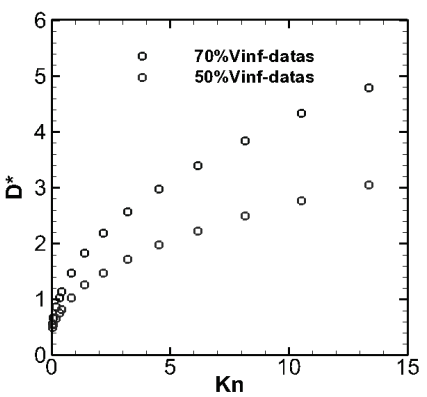

a. marked positions

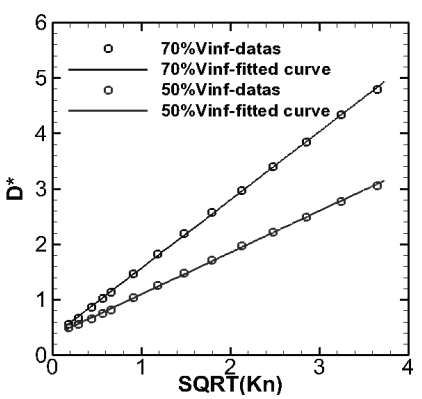

b. fitted curve

Fig. 4. Variation of perfect-gas flow with $K n$. 
According to the variation law discovered in this paper, an empirical method is established for perfect-gas flow field prediction at different Knudsen number in a given velocity. It is implemented as follows. First, two flow cases at different $K n$ in a given velocity are chosen as the fundamental cases and then simulated using the DSMC method. After that, marked positions are selected. According to the empirical formula $D^{*}=a \sqrt{K n}+b$, the two coefficients $a$ and $b$ can be calculated. Then, $D^{*}$ positions of flow field at another free-stream Knudsen number can be obtained. Changing the marked position and repeating the process, velocity distribution along the stagnation line at another $K n$ are plotted, instead of numerical simulation which costs a long time and computer resource. Thus, primary flow-field structure at different Knudsen number in a given velocity is predicted.

To establish the prediction method, thirteen cases covering the whole rarefied regime are simulated. As shown of the numerical results in Fig. 4b, all the marked points are distributed on the fitted curve. Analyzing from another aspect, if two of the thirteen cases are chosen as the basic cases, then the others all can be considered as the verification cases to the prediction method in this paper.

In the meanwhile, it is indicated that the empirical method can be used for the prediction of shock wave position. When $K n$ is close to zero, the free stream would become a continuum flow and $D^{*}$ tends to a constant number $b$ according to the empirical formula $D^{*}=a \sqrt{K n}+b$. In continuum regime, shock wave can be thought as a thin face, through which a supersonic flow turns to subsonic. If $D^{*}$ is located at an appropriate position $\left(M a^{*}=1 \sim M a_{i n f}\right)$, the coefficient $b$ can be considered as the predicted shock position.

\section{Analysis of $K n$ effects on chemical-reaction flow}

As we all know, hypersonic rarefied flows always contain high enthalpy along with high temperature effects. When temperature reaches an enough high value, some phenomena such as molecular dissociation and ionization may take place and there exist strong thermal-chemical non-equilibrium effects in flow field. Not only do chemical reactions have an enormous influence on aircrafts' aerodynamic forces and moments, transfer heating and radio communication, but also may cause a conspicuous difference in flow field relative to that with the perfect-gas model. So the effects of chemical reaction on hypersonic rarefied flow are investigated and variation law of flow field is reanalyzed.

\subsection{Free-stream conditions and computational grids}

For the comparison with the perfect-gas simulation cases, the same plate with a length $L=4 \mathrm{~m}$ and a thickness $t=0.05 \mathrm{~m}$ is used here. Three (the altitude $100,120,135 \mathrm{~km}$ ) of the cases in last section are recomputed. The $K n$ numbers are $0.035,0.83$ and 3.22. All the conditions except the gas model are kept identical, such as computational grids, freestream parameters and wall temperature. Five-species air chemical-reaction module presented in section II is introduced here. 


\subsection{Simulation results analysis}

To show the difference of chemical-reaction flow and perfect-gas flow clearly, flow properties including density (DEN), velocity (V) and overall temperature (TA) in both the two situations are illustrated in Figure 5 for the cases 100, 120 and $135 \mathrm{~km}$ from left to right. In the region $\mathrm{Y}>0$, there is chemical reaction taking place while there is none in $\mathrm{Y}<0$.

In the chemical-reaction region, a strong bow shock stands in front of the plate for the case $100 \mathrm{~km}$, through which overall density rises sharply due to the shock-layer compression. Shock gradually becomes weak and unapparent and flow field expands outside along with the increase of altitude and Knudsen number, which are similar to the change of perfect-gas flow characters. Figure 6 demonstrates the mole fractions of gas components in the three simulation cases, including $\mathrm{N}_{2}, \mathrm{O}_{2}, \mathrm{~N}, \mathrm{O}$ and $\mathrm{NO}$ along the stagnation line. We can clearly understand that there is no chemical reaction in the freestream region because the temperature is too low to have chemical reactions. The most significant chemical non-equilibrium process takes place in the case $100 \mathrm{~km}$. As seen in the picture, the amounts of $\mathrm{NO}, \mathrm{N}$ and $\mathrm{O}$ are increased while the other two are decreased when the flow passes through the bow shock due to the chemical reactions, which contain dissociation, exchange and recombination reactions. However, mole fractions of $\mathrm{N}_{2}$ and $\mathrm{O}_{2}$ rise up a little and the atoms fall down due to the cold wall effects. When the altitude increases, variation of each species along the stagnation is unapparent except the oxygen atom distribution around the wall. It is shown that chemical non-equilibrium effects are weakened due to the low inflow density. And there aren't enough molecular collisions to cause dissociation of nitrogen and oxygen molecules happening though the temperature is relatively high.

Comparing the two situations chemical reaction and no chemical reaction, flow field shows the apparent differences. Shock wave in the former cases is stronger and closer to the wall surface and flow-field structures are compressed more intensely than the latter, which is presented more clearly in velocity distributions along the stagnation line in Figure 7a. It is mainly caused by the high temperature gas effects and chemical nonequilibrium effects. Figure $7 \mathrm{~b}$ is the overall temperature distributions along the stagnation line. The temperatures in the former cases are higher than the latter. Taking the $100 \mathrm{~km}$ case for example, the peak temperature after the shock wave changes from approximately $26000 \mathrm{~K}$ for perfect gas to around $18000 \mathrm{~K}$ when considering the chemical reactions, which shows an obvious chemical non-equilibrium process right after the shock wave. However, these differences decrease along with the altitude rising up. The differences in the $135 \mathrm{~km}$ case are too slight to be ignored as the computation errors due to the lowdensity effects and weak chemical reactions as shown in Figure 6c. 


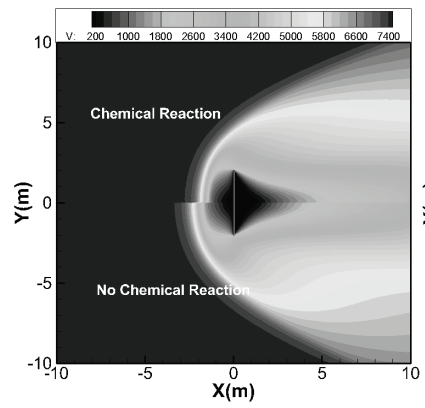

a. $100 \mathrm{~km}$

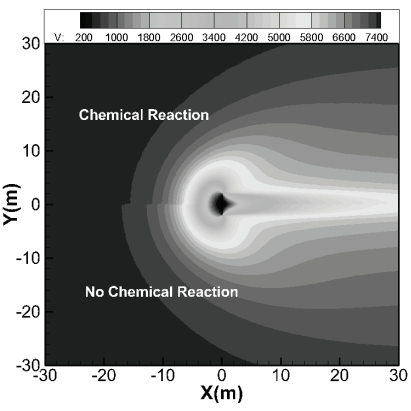

b. $120 \mathrm{~km}$

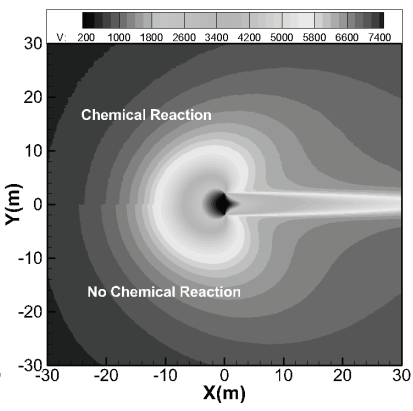

c. $135 \mathrm{~km}$

Fig. 5. Velocity contours comparison between the chemical-reaction flow and perfect-gas flow.

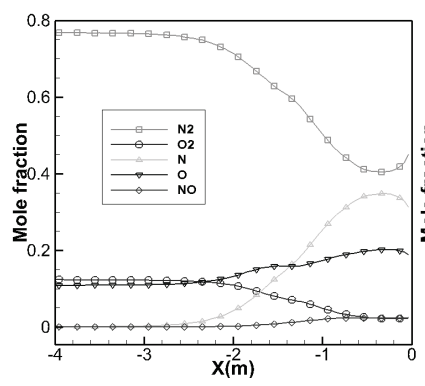

a. $100 \mathrm{~km}$

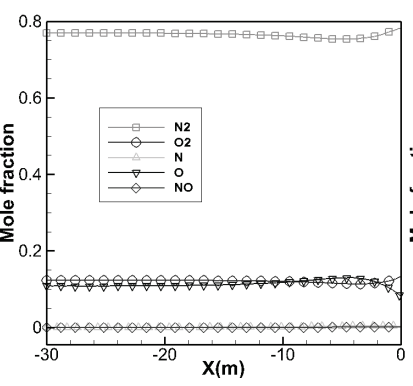

b. $120 \mathrm{~km}$

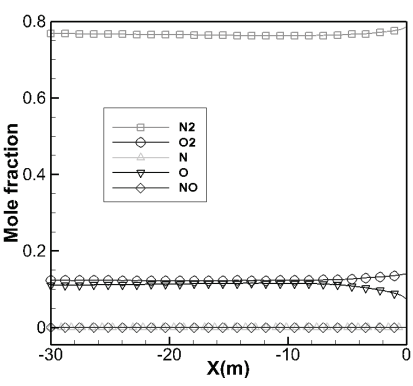

c. $135 \mathrm{~km}$

Fig. 6. Mole fractions of gas components along the stagnation line.

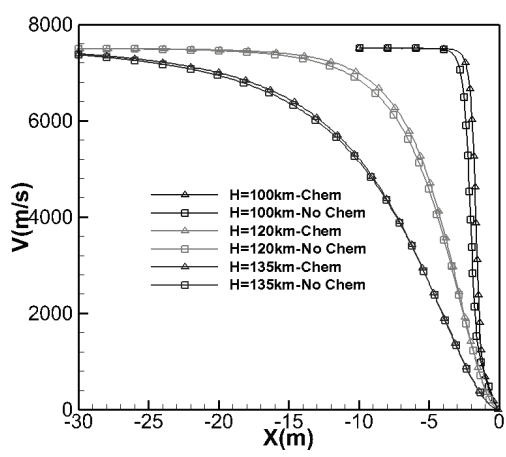

a. velocity

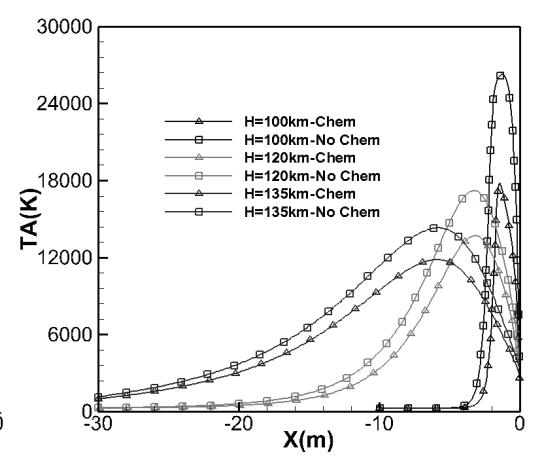

b. overall temperature

Fig. 7. Velocity and overall temperature distributions along the stagnation line.

\subsection{The empirical method in chemical-reaction method flow}

As presented in subsection 4.2, flow field is changed a lot when considering chemical reactions, including the structures, shock wave stand-off distance and flow properties and 
so on. Therefore, the empirical method discovered in perfect-gas flow need to be verified in chemical-reaction conditions.

Using the same analysis method in section 3, we choose the dimensionless positions of two velocities $50 \% V_{\text {inf }}$ and $70 \% V_{\text {inf }}$ in the stagnation line as the markers. In Figure 8 , the dashdotted lines show the fitted line of perfect-gas flow field scale as a function of $K n$ based on the least-square method, which are part of the lines in Figure 4b. The square and triangle symbols represent distribution of the marked positions versus $K n$ in chemicalreaction flow. As seen in the picture, the flow field still expands outside linearly with $\sqrt{K n}$ increasing when considering chemical reactions. However, the expansion tendency is sharper than the perfect-gas flow with no chemical reactions.

Based on the analysis, the empirical formula $D^{*}=a \sqrt{K n}+b$ is verified and the method for fast prediction of flow-field structure can be used in chemical-reaction flow.

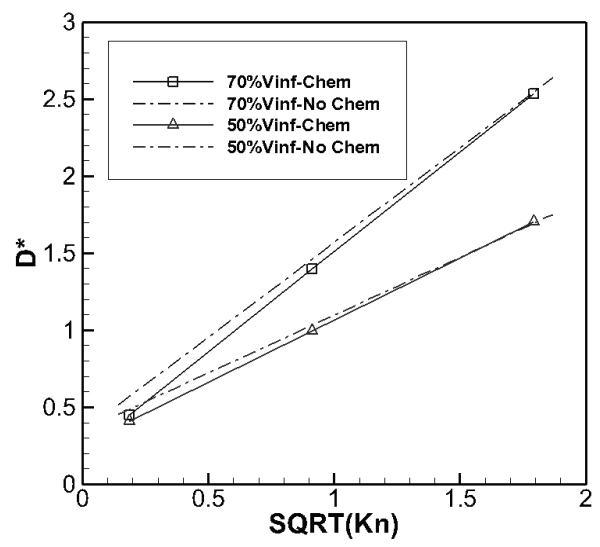

Fig. 8. Variation of chemical-reaction flow with $K n$.

\section{Conclusions}

In this paper, the effects of the free-stream Knudsen $(K n)$ number on hypersonic rarefied flow-field structure are studied numerically based on the unstructured DSMC method. First, the vertical-plate flows under perfect-gas conditions at different $K n$ numbers are simulated. Then, the effects of chemical reactions on flow-field structure are researched by introducing five-species chemical module and the variation of chemical-reaction flow field as a function of $K n$ is analyzed. The conclusions are presented as follows. The rarefied flow-field structure is compressed more strongly and flow properties are changed a lot when considering chemical reactions. However, no matter with the perfect-gas model or the chemical-reaction model, the flow field both expands outside linearly as a function of $\sqrt{K n}$ in a given inflow velocity shown as an empirical formula $D^{*}=a \sqrt{K n}+b$, but the expansion tendency is a little sharper in the latter situation. Then, the empirical method for fast prediction of rarefied flow field is proposed, through which the cases in a given velocity at different $K n$ can be estimated instead of numerical simulation. 
However, in this work, the simulation geometry is limited to a two-dimensional vertical plate with no other geometries considered. Based on the variable control principle in research, the free-stream conditions except molecular number density are kept the same, for example, the velocity state, the gas components and inflow temperature. And the effects of these conditions on the flow-field variation law versus $K n$ are not studied in this paper. Therefore, the empirical method need to be verified further more in future's work about the application, the suitability, the influence factors, and so on.

\section{References}

1. She Q., Rarefied Gas Dynamics[M], in National Defence Industry Press(Beijing, 2003).

2. Tsien H. S., Superaerodynamics, Mechanics of Rarefied gases, J. Aeronaut. Sci., 13(12), (1964), 653-664.

3. Bird G.A., Molecular Gas Dynamics, in Clarendon Press, (Oxford, UK, 1976).

4. Bird G.A., Molecular Gas Dynamics and the Direct Simulation of Gas Flow[M]. Oxford: Clarendon Press (1994), p. 306 310.

5. Fan J., Rarefied gas dynamics: Advances and applications, Advances in Mechanics, 43(2), 2013, 185 201 (in Chinese).

6. Bird G.A., Application of the DSMC method to the full shuttle geometry, AIAA-Paper 901692, 1990.

7. Moss J.N., Wilmoth R.G., Price J.M., DSMC simulation of blunt body flows for mars entries: Mars Pathfinders and Mars microprobe capsules, AIAA-97-2508, 1997.

8. Boyles K.A., LeBeau G.J., DSMC Simulation in Support of the Columbia Shuffle Orbiter Accident Investigation, AIAA 2004-2282, 2004.

9. Moss J.N., DSMC Simulation of Ballute Aerothermodynamics Under Hypersonic Rarefied Conditions, AIAA 2005-4949, 2005.

10. Sun Q., Fan J., Liu H., Jiang J., Rarefied Gas Effects On The Aerodynamics Of Hypersonic Air Vehicles, in The first session of the Hypersonic Technology Conference (Yunnan, China, 2008) (in Chinese).

11. Ozawa T., Takayanagi H., Suzuki T., Fujita K., Analysis of Rarefied Aerodynamic Effects for Mars Entry Missions, AIAA 2012-3101, 2012.

12. Potter J.L., Peterson S.W., Local Bridging to Predict Aerodynamics Coefficients in Hypersonic, Rarefied Flow, in Journal of Spacecraft and Rockets, Vol. 29, No. 3, 1992: 344 351.

13. W. X., W. Y., X. J., The implementation of unstructured DSMC method for 2D thermodynamics and chemical reaction non-equilibrium flow and its application, in ACTA Aerodynamics Sinica, Vol.24, No.2, 2006 (in Chinese).

14. Gupta R.N., Yos J.M., Thompson R.A., Lee K.P., A Review of Reaction Rates and Thermodynamics and Transport Properties for an 11-Species Air Model for Chemical and Thermal Nonequilibrium Calculations to $30000 \mathrm{~K}, N A S A-R P-1232,1990$.

15. Anon. U.S., Standard Atmosphere, 1976, National Oceanic and Atmospheric Administration, NASA and U.S. Air Force, Oct. 1976. 